\title{
Roles of Galectin-7 in Cancer
}

\section{Manpreet Kaur ${ }^{1}$, Tarnjeet Kaur ${ }^{1}$, Sukhdev Singh Kamboj² ${ }^{2}$ Jatinder Singh ${ }^{2 *}$}

\begin{abstract}
Galectins are $\beta$-galactoside binding lectins that contain one or more carbohydrate recognition domains. As a consequence of sugar-binding properties, galectins exhibit a variety of interactions with glycoproteins, thus playing important roles in various pathological processes. A number of studies have shown roles of galectins in cancer. Galectin-7 is a prototype member of the galectin family implicated in epithelial stratification and cell migration. It can act as a potent dual regulator in different types of cancer. Galectin-7 may contribute either to neoplastic transformation and tumour progression through regulation of cell growth, cell cycle, angiogenesis, apoptosis and cell migration or may have a protective effect in cancer depending on the tissue type. A perusal of the literature indicates particular roles of galectin-7 in carcinomas and melanomas, while contributions await greater exploration in other types of cancers including sarcomas and leukemia. This review collectively summarizes available literature on expression and roles of galectin-7 in different cancers.
\end{abstract}

Keywords: Carcinoma - galectin - lectin - lymphoma - Metastasis

Asian Pac J Cancer Prev, 17 (2), 455-461

\section{Introduction}

Lectins are non-enzymatic and non-immunoglobulin proteins that bind carbohydrates with high specificity. Galectins (previously called S-type lectins) are $\beta$-galactoside binding lectins that contain one or more carbohydrate recognition domains (Barnodes et al., 1994). Currently there are 15 mammalian galectins known, 11 of which are found in humans, namely galectins 1-4, 7-10, 12, 13 and 14. Depending on structural organization, galectins are classified as prototype, tandem repeat type and chimera type galectins. Prototype galectins have only one carbohydrate recognition domain (CRD) and exists as monomers or non-covalent dimers. Tandem repeat type galectins are characterized by two non-identical CRDs, which are linked by a short linker peptide of up to 70 amino acids while chimera type galectin has both CRD as well as N-terminal domain (Leffler et al., 2004). Galectin-7, a member of prototype galectin family, is encoded by gene LGALS7 located on chromosome 19q13.2. Like other members of galectin family, galectin-7 lacks a signal peptide sequence required for its secretion and thus can be secreted via non-classical pathway of secretion (Hughes 1999). Galectin-7 can bind to a large number of potential receptors as that of other galectins including non-reducing terminal $\mathrm{N}$-acetyl lactosamine (LacNac) residues as well as to internal LacNac oligosaccharide residues (Brewer 2004). It exhibits a high degree of tissue specificity and is specifically expressed by keratinocytes (Magnaldo et al., 1995). Its expression was found to be restricted mostly to stratified epithelial cells of the oesophagus, tongue, lip and epidermis, the oral epithelia, the cornea, Hassall's corpuscles of the thymus, the urinary system, the stratified squamous epithelium of the stomach and mammary myoepithelial cells (Magnaldo et al., 1998; Sato et al., 2002; Saussez and Kiss, 2006).

The extensive literature indicated the role of galectins in different types of cancer (Satelli et al., 2008; Balan et al., 2010; Vladoiu et al., 2014). The etiology of cancer involved the complex, assorted, distinct biological processes. Altered glycosylation and defects in apoptosis are the hall marks in pathogenesis of cancer. So understanding the significance and biological functions of altered glycosylation may serve the purpose of unfolding the key events involved in tumour pathogenesis. Galectins exhibit a variety of cell-cell and cell-matrix interactions and showed high affinities for specific oligosaccharides which make these molecules promising markers as well as therapeutic targets for studying their role in etiology of cancer. During the past few decades the pro/anti- apoptotic role of galectins $1,-3,-9$ in cancers had been extensively reviewed (Liu et al., 2005; Balan et al., 2010; Newlaczyl and Yu., 2011). In the last decade a number of studies had explored the role of galectin-7 in cancer. It may contribute either to neoplastic transformation and tumour progression through the regulation of cell growth, angiogenesis, apoptosis and cell migration or have a protective effect in cancer depending on tissue type of cancer. The varied expression of galectin-7 was extensively studied in carcinoma type of cancer, while very few studies had been reported in other types of cancers.

The focus of the present review is to provide for the 


\section{Manpreet Kaur et al}

first time, the comprehensive literature on the role of galectin-7 in different types of cancers and its prospects as a diagnostic marker and therapeutic target for the same.

\section{Galectin-7 in Carcinomas}

\section{Breast cancer}

Various studies had indicated the increased expression of galectin-7 in breast cancer. Galectin-7 mRNA was found to be over-expressed in a rat model of chemically induced mammary carcinoma but not in colon adenocarcinoma. This finding suggested galectin-7 as one of the mammary carcinoma specific molecular marker (Lu et al., 1997). A later study using preclinical mouse models also indicated high expression of galectin-7 in breast cancer cells (Demers et al., 2010). This over-expression of galectin-7 was found to increase the metastatic behavior of these cells, rendered them resistant to apoptosis. Furthermore, elevated galectin-7 expression was found to be restricted to high grade carcinomas, over expressing human epithelial growth factor receptor-2 (HER-2) as well as basal like groups. Based on these findings, galectin-7 was proposed as a specific marker for these two types of mammary carcinomas as well as a metastatic marker.

The over-expression of galectin-7 in breast cancer as well as its ability to increase its metastatic behavior was contradictory to the fact that galectin-7 gene was well documented as a p53 induced gene (Polyak et al., 1997). This paradox was addressed by some recent studies. Transfection of breast cancer cells with expression vectors encoding mutant p53 was shown to induce galectin-7 expression at both mRNA and protein levels, even after doxorubicin, an anti cancer drug, treatment. However, knockdown of endogenous mutant p53 inhibited doxorubicin induced galectin-7 expression. A positive correlation of p53 induced galectin-7 expression has been observed with increased nuclear factor-kB (NF$\mathrm{kB}$ ) activity in breast cancer cells and the expression was inhibited by NF-kB inhibitors also. These findings suggested that p53 induced expression of galectin-7 was NF- kB dependent. Galectin-7 promoter was also shown to harbor a NF-kB binding site. TNF- $\alpha$ induced galectin-7 expression was also inhibited by NF-kB inhibitors (Campion et al., 2013). These findings suggested galectin-7 to be part of the common pathway used by mutant p53 to promote cancer progression. Ectopic expression of CCAAT/enhancer binding protein $\beta-2(\mathrm{C} /$ EBP $\beta-2)$, another transcription factor, in human breast cancer was shown to induce expression of galectin-7 at both mRNA and protein levels (Campion et al., 2014). In silico analysis further revealed $\mathrm{C} / \mathrm{EBP} \beta-2$ binding site in promoter region of galectin-7 and mutation in this site was shown to abolish the transcriptional activity of galectin-7 promoter. Furthermore, binding of endogeneous galectin-7 promoter with $\mathrm{C} / \mathrm{EBP} \beta-2$ was also shown by chromatin immuno-precipitation. Immuno-histochemical studies also revealed the similar expression pattern of $\mathrm{C} / \mathrm{EBP} \beta-2$ and galectin-7 in myoepithelial and basal like breast cancer cells. These findings suggested that galectin-7 expression with mutant p53 was possibly mediated by NF-kB and $\mathrm{C} / \mathrm{EBP} \beta-2$, and thus giving the plausible explanation for anti-apoptotic behavior of galectin-7 in breast cancer.

The resistant of breast cancer cells to apoptosis is argued to be dependent on cellular localization of galectin-7. To demonstrate this, MCF-7 breast carcinoma cell line was transfected with a mutant form of galectin7(R74S) with altered sub-cellular location (Grosset et al., 2014). Normally intracellular galectin-7 was found to be expressed in cytoplasm, nucleus and mitochondria. Mutation at position 74 affected the translocation of galectin-7 to mitochondrial and nuclear compartments and also reduced the affinity to bind with glycoproteins. Both types of cells including those harbouring wild type galectin-7, as well as mutant, were found to be equally resistant to drug induced cell death, demonstrating the anti-apoptotic property of galectin-7 irrespective of its intracellular localization. Cells expressing R74S were even more resistant to apoptosis induced by etoposide and doxorubicin treatment. A recent study further showed that both wild type as well as mutant forms of galectin-7 had equal affinity for bcl-2 under in-vitro as well as in-vivo conditions (St-Pierre et al., 2015).

As dox-induced DNA damage was documented to increase $\mathrm{p} 21$ expression via $\mathrm{p} 53$ dependent pathway, the expression of p21 and p53 was also studied in MCF-7 cells. These results indicated the decreased expression of both of these molecules in wild type galectin-7 and R74S cells. The inhibition observed in R74S cells was even stronger than the cells with wild type form of galectin-7 (Grosset et al., 2014). So these recent findings challenges the current trends of targeting mitochondrial galectin-7 and it has been proposed that CRD independent cytosolic galectin-7 irrespective of its mitochondrial expression could be selectively targeted for treatment of breast cancer cells.

\section{Head and neck squamous cell carcinoma}

Many studies had suggested galectin-7 as potent marker in head and neck squamous cell carcinoma (HNSCC). Significantly high expression of galectin-7 was observed in squamous cell carcinoma of buccal mucosa and oesophagus (Chen et al., 2004; Zhu et al; 2010). High expression of galectin-7 was also reported in tongue squamous cell carcinoma which was significantly correlated with different histological grades of tumour (Alves et al., 2011). Proteomic analysis indicated elevated expression of galectin-7 in nasal inverted papilloma (Meng et al., 2010). Immuno-histochemical studies also revealed distinctive expression of galectin-7 in inverted papillomas as compared to other non-malignant nasal pathologies like chronic rhinosinusitis and nasal polyposis (Duray et al., 2014).

In a series of papers, Saussez et al reported various expression studies of galectin-7 in squamous cell carcinoma of head and neck. Immuno-histochemical study revealed expression of galectin-7 in stage IV hypopharyngeal squamous cell carcinoma. No significant association of galectin-7 expression was found with tumour staging and metastatic status. On the other hand, the expression was significantly associated with histological differentiation of tumour and rapid recurrence rate after surgery (Sauseez et al., 2006). However, no detectable serum galectin-7 levels 
were observed in hypopharyngeal cancer patients (Saussez et al., 2008b). Furthermore, expression of galectin-7 was found to vary in different grades of tumour (Saussez et al., 2008a). The highest expression of galectin-7 was observed in hypopharyngeal and larynx SCCs followed by high grade dysplasia, low grade dysplasia and normal epithelia indicating that galectin-7 may lead to cancer progression. In addition, increased expression of galectin-7 in different grades of dysplasia to carcinomas was accompanied by a change in its cellular location i.e. from the nucleus to the cytoplasm. Tumour progression in head and neck squamous carcinoma is also being affected by sub-cellular localization. In oesophageal cancer galectin-7 was found to be expressed in cytoplasm, nucleus and membranes of cells, while in normal oesophageal tissues its expression was restricted in nucleus only (Zhu et al., 2010).

Another study also reported high expression of galectin-7 in squamous carcinoma of head and neck, but not in basal cell carcinoma (Cada et al., 2009). This higher expression was not related to tumour and lymph nodes staging (TN staging), but significantly correlated with high degree of differentiation and keratinization. Significant positive correlation of galectin-7 and matrix metalloproteinases-9 (MMP-9) had been observed in laryngeal squamous cell carcinoma, indicating plausible potential role of galectin-7 as inducer of MMP-9 gene expression in-situ (Saussez et al., 2009). High expression of galectin-7 had been observed in oral epithelial dysplasia as compared to normal oral mucosa. Comparison of galectin-7 expression according to epithelial localization of oral epithelial dysplasia (OED) showed the elevated expression in upper /middle in most of the OED (de Vasconcelos Carvalho et al., 2013). Recent study on oral SCC showed that galectin-7 can act as potential predictive marker of chemo/radio therapy resistance in this cancer. Oral SCC cell line showed decreased cell viability with elevated expression of galectin-7 (Matsukawa et al., 2014).

\section{Cervical cancer}

Role of galectin-7 had been implicated in squamous and adenocarcinoma types of cervical cancer. Proteomic analysis showed the differential expression of various proteins associated with concurrent chemo-radiotherapy (CCRT) response. Out of the various proteins involved, elevated galectin-7 expression was significantly found to be associated with increased sensitivity towards CCRT. It was postulated that this protein could not be used as independent predictive factor for sensitivity towards CCRT. Galectin-7 along with other proteins like S100A9, NMP-238 and HSP-70 provided a full recurrence prediction model towards CCRT response (Zhu et al., 2009). Another study provided the experimental data to further explore the role of galectin-7 along with S100A9 in cervical cancer. S100A9 is documented as a novel p53 transcriptional target, which induces cell apoptosis via p53 dependent pathway. The syngerstic as well as independent effects of both these proteins with clinocopathological variables had been studied in cervical squamous cell carcinoma (Zhu et al., 2013). Immuno-histochemical studies revealed the reduced expression of galectin-7 and S100A9 in cervical squamous carcinoma. The galectin-7
Expression and Roles of Galectin-7 in Different Types of Cancer was found to be expressed in both cytoplasm and nucleus, while on the other hand S100A9 expression was mainly observed in nucleus. Positive staining of cervical carcinoma tissues with galectin-7 and S100A9 was shown to be negatively associated with lymph nodes metastasis, staging of cancer and MMP-9 expression. Differentiation potential of cervical carcinomas was found to be directly correlated with galectin-7 expression. However, syngerstic effect of both proteins showed much higher effect on overall survival (OS) rate. Also in-vitro studies revealed that knock down of galectin-7 and S100A9 increased MMP-9 expression and activation of PI3K/Akt signaling pathways, which resulted in increased cell viability and cell proliferation under induced stress conditions (Zhu et al., 2013). Galectin-7 expression was also found to affect the OS rate in cervical squamous carcinoma as indicated by Kaplan -Meier survival analysis. Negative staining of both proteins in cervical carcinoma patients, independently as well as synergistically showed significantly lower 5 year over OS rate as compared to tissues with positive staining as indicated by Kaplan -Meier survival analysis. This finding was further supported by the evidence that elevated galectin-7 expression had been associated with improved outcomes after radiation therapy (Tsai et al., 2013). All these findings attributed towards protective effect of galectin-7 in cervical squamous cell carcinoma.

Besides squamous cell carcinoma, the contrasting role of galectin-7 had also been documented in adenocarcinoma of cervix (Park et al., 2009). Human cervix epithelial cells (HeLa) transfected with galectin-7 were shown to increase MMP-9 expression as compared to non-transfected HeLa cells. Increased MMP-9 expression was resulted in increased metastatic potential of HeLa cells in a matrigel invasion system. This induced MMP-9 expression by galectin-7 might be regulated through p38 signaling pathways, as increased phosphorylated p38 levels have been observed on transfection with galectin-7. This finding was further supported by the evidence that inhibition of p38 MAPK was shown to reduce levels of galectin-7 induced MMP-9 expression.

\section{Bladder cancer}

Majority of bladder cancers are urothelial cell carcinomas $(90 \%)$ while squamous cell carcinomas constitute only $10 \%$ of bladder cancers. Galectin-7 expression varied in different types of bladder cancers. Elevated expression of galectin-7 had been observed in highly differentiated squamous cell carcinoma, while urothelial carcinoma showed reduced galectin-7 expression as compared to respective normal cells (Ostergaard et al., 1997; Matsui et al., 2007). Elevated expression of galectin-7 had been observed in more differentiated tumours as compared to less differentiated tumour (Ostergaard et al., 1997).

An in-vitro study on urothelial carcinoma documented the induced expression of galectin-7 on exposure to cisdiamminedicholroplatinum (CDDP) in urothelial cancer cell lines with wild type p53 but not in with mutant p53. Increased sensitivity towards CDDP had been observed in galectin-7 transfected cells (with mutant p53) as compared to control transfectants. In addition to this, accumulation 
Manpreet Kaur et al

of intracellular reactive oxygen species (ROS) and activation of JNK and Bax had been observed in galectin-7 transfected cells than control transfectants. Based on these findings it was proposed that galectin-7 might act as predictive marker of chemo-sensitivity against CDDP (Matsui et al., 2007).

\section{Ovarian cancer}

Immuno-histochemical studies revealed the elevated expression of galectin-7 on epithelial ovarian cancer cells (Kim et al., 2013a; Labrie et al., 2014). On the other hand, no detectable levels have been observed in normal ovarian tissue. In-vitro studies revealed that the expression of galectin-7 in ovarian carcinoma was being regulated by p53 mutants than wild type 53. Down-regulation of p53 mutant using siRNA further provided evidence for this finding. Furthermore, elevated galectin-7 expression was found to be positively correlated with histological grades of tumour, advanced age, high mortality rate and poor survival outcome (Kim et al., 2013a; Labrie et al., 2014). The galectin-7 was predominantly expressed in nucleus in ovarian cancer (Kim et al., 2013a). On contrary, another study indicated the galectin -7 was mainly localized in cytoplasm and extracellular compartment (Labrie et al., 2014). In-silico analysis further confirmed the significant association of galectin-7 with survival outcome in ovarian cancer adeno-carcinoma (Labrie et al., 2014).

Galectin-7 expression was also shown to increase the metastatic behavior of ovarian cancerous cells (Labrie et al., 2014). Matrigel invasion assays and live cell imaging showed that galectin-7 increased the invasive behavior of ovarian cancer cells by inducing MMP-9 and increasing cell motility. Another study further confirmed that siRNA induced galectin-7 down-regulation resulted in inhibition of cellular proliferation of an ovarian cell line A2780-PAR (Kim et al., 2013a). Like other galectins, galectin-7 is documented as a key mediator in tumour metastasis mediating through induction of immuno-suppression within tumour microenvironment. Recombinant human gal-7 resulted killing of Jurkat T cells and human peripheral T cells, suggesting that galectin-7 also has immunosuppressive properties. Taken together, these findings can provide a new rationale for targeting galectin-7 in epithelial ovarian cancer.

\section{Gastric and colon cancer}

In-vitro as well as in-vivo studies had indicated tumour suppressive effect of galectin-7 on colon cancer (Ueda et al., 2004). Colon carcinoma cell line transfected with galectin-7 (DLD 1-gal-7) had been shown to have slower growth rate in comparison to control transfected cells (DLD 1-V). Furthermore, ectopic expression of galectin-7 rendered the colon carcinoma cells more sensitive towards apoptosis under different stimuli like treatment with actinomycin D, cobalt chloride, hydrogen peroxide or hypoxic condition. Significant reduction in tumour growth had been observed in SCID mice on inoculation with DLD 1-gal-7 cells in comparison to DLD 1-V cells. Furthermore, DLD 1-gal-7 inoculated mice showed lower proliferation rate as well as reduced number of blood vessels, suggesting that ectopic expression of galectin-
7 suppresses angiogenesis. This may partially account for the greater suppressive effect of galectin-7 on tumor growth in vivo than in vitro angiogenesis in comparison to control cells.

Reduced expression of galectin-7 had been reported in gastric cancer tissues as compared to normal tissues from patients. The galectin-7 expression was mainly observed in cytoplasm (Kim et al., 2013a). This, reduced expression was also significantly associated with advanced TMN stage (tumour, metastasis, nodes) of disease and better survival rate of gastric cancer patients. The transfection of AGS cells, a gastric cell line, with galectin-7 expression vector led to the suppression of cell proliferation, migration and tumour invasion. On the other hand, knockdown of galectin-7 in KATO III gastric carcinoma cells reversed these attributes. Null tumour growth on inoculation of galectin-7-overexpressing AGS cells into nude mice, suggesting suppressive role of galectin-7 in gastric cancer.

Further, galectin-7 expression was found to be regulated by DNA methylation. In-vitro study indicated aberrant methylation of galectin-7 gene in gastric cancer cells compared to normal cells. This finding was further strengthened by the fact that treatment of gastric cancer cells with aza-deoxycytidine, a demethylating agent, resulted in elevated galectin-7 mRNA expression (Kim et al., 2013a). Taken together these findings conferred the role of DNA methylation in regulation of galectin-7 expression.

\section{Thyroid cancer}

Galectin-7 expression was found to vary in different types of thyroid tumour. Immuno-histochemical studies revealed higher percentage of galectin-7 positive cells as well as its elevated expression in carcinomas in comparison to adenomas (Rorive et al., 2002; Than et al., 2008). In contrast, immuno-histochemical study showed no significant difference in galectin-7 expression between benign and malignant forms of thyroid cancer (Than et al., 2008). Furthermore, up-regulated differential expression of galectin-7 and cytokeratin-19, an established marker in thyroid cancer, in papillary carcinomas as compared to micro-follicular adenomas has been reported. So based on these findings, it was proposed that galectin-7 along with cytokeratin-19 can act as a diagnostic biomarker to differentiate between benign and malignant forms of thyroid cancer.

\section{Galectin-7 in Lymphomas}

Initial study reported the differential expression of 1176 known genes in S19, an aggressive lymphoma cell line and 164T2 a non-aggressive lymphoma cell line (Moisan et al., 2003). Out of the various genes reported, the constitutive expression of galectin-7 has been observed in aggressive metastatic variants of lymphoma cells. Furthermore, western blot analysis and immunehistochemical study revealed the induced galectin-7 expression in several organs of murine model. Also, expression of galectin-7 was induced at early stages of tumour progression and it was independent of tumour 
microenvironment.

In a series of papers, this group of authors further reported the role of galectin-7 in $\mathrm{T}$ cell lymphoma. Transfection of galectin-7 cDNA in lymphoma cells of murine model showed significant difference in lymphoma growth rate as indicated by survival mean time of mice as compared to control mice (Demers et al., 2005). On the other hand, galectin-7 transfected 267 $\mathrm{T}$ lymphoma cells showed no significant differences in growth rate in comparison of control cells. Furthermore, histopathological analysis in galectin-7 transfected murine model revealed the development of large metastatic tumours with massive infiltration of tumor cells in the parenchymas in both lymphoid as well as non-lymphoid cells. Transfection of galectin-7 in non-aggressive lymphoma cells of ICAM-deficient mice were also shown to increase metastatic potential of these cells as compared to control cells. Galectin-7 transfectant cells were further shown to have elevated MMP-9 expression as compared to control transfectants (Demers et al., 2007). This induced elevated MMP-9 expression was found to be galectin-7 specific, as its expression was completely abolished on addition of $\beta$-lactose. All these findings collectively indicated that galectin-7 can modulate the malignant growth mediated via MMP-9 expression under in-vivo conditions. This group of authors further conducted an in-vivo experiment to target galectin-7 with antisense strategies in lymphoma cells. The transfection of lymphoma cells with plasmid encoding anti-sense galectin-7 cDNA led to reduced MMP-9 expression, tumourigenicity and markedly inhibited tumour metastasis (Demers et al., 2007).

Studies indicated the higher expression of galectin-7 in different cancers including lymphoma. Up-regulated expression of galectin-7 in lymphoma cells was certainly not attributed to the p53 gene, which was previously thought to be the inducer of galectin-7 (Demers et al., 2009). This finding was evidenced from the fact that treatment of lymphoma cells with doxorubicin was sufficient to induce p53 expression, but de-novo expression of galectin-7 was not observed in those cells. Induced expression of p53 dependent gene, p21 in doxorubicin treated cells further confirmed the functional potential of
Expression and Roles of Galectin-7 in Different Types of Cancer p53 gene. All these findings directed towards any another mechanism which modulate the expression of galectin- 7 . Epigenetic regulation is the one of the mechanism which was thought to control the expression of different galectin family members (Benvenuto et al., 1996; Ruebel et al., 2005). DNA methylation was proposed to be associated with aberrant expression of galectin-7 in lymphoma cells. In-vitro study indicated the hyper-methylation of CpG sites in cells expressing lower or null galectin-7 expression. On the other hand, cells expressing elevated galectin-7 levels were found to be having hypo-methylated CpG sites. Furthermore, different studies also reported the induction of galectin-7 expression in human and murine lymphoma cells on treatment with 5-aza-2'-deoxycytidine, a demethylating drug (Moisan et al., 2003; Demers et al., 2009). Taken together, all these findings proposed the role of aberrant methylation in regulation of galectin-7 gene expression in malignant form of lymphomas.

Besides T-cell lymphoma, up-regulated expression of galectin-7 had also been observed in B-cell neoplasmas (Demers et al., 2007).

\section{Galectin-7 in Melanomas}

Differential expression of multiple proteins have been observed on treatment of mouse skin on treatment with cypermethrin, a synthetic pyrethroid insecticide; benzo[a]-pyrene, tumor initiator; 12-O-tetradecanoyl-phorbol13-acetate, stage I tumor promoter and mezerein stage II tumor promoter. Up-regulated expression of galectin-7 has been observed in response to above said carcinogens induced melanomas (George et al., 2011). Different studies further indicated the up-regulated expression of galectin-7 in transgenic mice models (BK5.IGF-1, K14.COX-2 TG)) having genes for insulin-like growth factor-1(IGF-1) and cyclooxygenase-2 (COX-2), indicating its role in IGF1 and COX-2 induced melanomas (Shen et al., 2007a; Shen et al., 2007b). In contrast, another study reported lower expression of galectin-7 in benign and malignant melanoma patients compared to controls and patients with nevi as indicated by in-silico analysis (Biron-Pain et al., 2013). Study involving B16F1, a melanoma cell line, confirmed galectin-7 expression at tumour site and

\section{Table 1. Differential Expression and Function of Galectin-7 in Cancer}

\begin{tabular}{|c|c|c|}
\hline Type of cancer & Cellular localization & Expression and effect \\
\hline \multicolumn{3}{|l|}{ A. Carcinomas } \\
\hline Breast cancer & Cytoplasm, Nucleus, Mitochondria & \multirow{2}{*}{$\begin{array}{l}\text { Elevated expression, resistance to apoptosis, induces chemoresistance } \\
\text { Increased expression, positive correlation with MMP-9, predictive marker } \\
\text { of chemo/radio therapy resistance }\end{array}$} \\
\hline Head and Neck Squamous cell carcinoma & a Nucleus & \\
\hline \multicolumn{3}{|l|}{ Cervical cancer } \\
\hline a. Adeno-carcinomas & Nucleus & Elevated expression, Invasive behavior \\
\hline b. Squamous cell carcinoma & Nucleus, cytoplasm & $\begin{array}{l}\text { Reduced expression, galectin- } 7 \text { knockdown showed increased invasion, } \\
\text { increased sensitivity towards CCRT }\end{array}$ \\
\hline \multicolumn{3}{|l|}{ Bladder cancer } \\
\hline a. Squamous cell carcinoma & \multirow[t]{2}{*}{ Not documented } & Elevated expression \\
\hline b. Urothelial carcinoma & & Reduced expression \\
\hline Ovarian cancer & & Elevated expression, increased metastasis \\
\hline Gastric and colon cancer & \multirow{2}{*}{ Cytoplasm, nucleus } & Reduced expression, reduced cell growth, low proliferation rate \\
\hline Thyroid cancer & & Elevated expression in adenomas as compared to carcinoma \\
\hline B. Lymphomas & Not documented & $\begin{array}{l}\text { Elevated expression in both T cell lymphoma and B cell neoplasmas, } \\
\text { increased MMP-9 expression }\end{array}$ \\
\hline C. Melanomas & Not documented & Elevated expression, role in metastasis \\
\hline
\end{tabular}




\section{Manpreet Kaur et al}

its role in metastasis. Furthermore, elevated galectin-7 expression rendered $\mathrm{B} 16 \mathrm{~F} 1$, a melanoma cells resistant towards apoptosis and reduced their cellular motility via induced EGR-1 expression. However, when B16F1 cells were injected into mice, elevated galectin-7 expression was failed to modulate the tumour growth.

The role of galectin-7 in different cancers with its differential expression and sub-cellular localization has been summarized in Table 1.

\section{Concluding Remarks}

A perusal of literature indicates that like other galectins, galectin-7 also act as positive or negative regulator of tumour growth and progression. This behavior can be attributed to the complex biological interactions as well as their compartmentalization in different cellular types or might be due to tumour heterogeneity. So, targeting a molecule depending on its sub-cellular localization in a particular type of cancer may provide a new insight for the development of anticancer drugs with improved efficacy. But more in-depth studies with detailed elucidation of molecular actions of galectin-7 in tumour progression are required to being viewed it as potential diagnostic and therapeutic target in cancer.

\section{Acknowledgements}

This work was supported by the grant of University Grants Commission (UGC), New Delhi, India [F.1774/2008(SA1)].

\section{References}

Alves PM, Godoy GP, Gomes DQ, et al (2011). Significance of galectins- $1,-3,-4$ and -7 in the progression of squamous cell carcinoma of the tongue. Pathol Res Pract, 207, 236-40.

Balan V, Nangia-Makker P, Raz A (2010). Galectins as cancer biomarkers. Cancers (Basel), 2, 592-10.

Barondes SH, Cooper DN, Gitt MA, Leffler H (1994). Galectins:Structure and function of a large family of animal lectins. J Biol Chem, 269, 20807-10.

Benvenuto G, Carpentieri ML, Salvatore P, et al (1996). Cell-specific transcriptional regulation and reactivation of galectin-1 gene expression are controlled by DNA methylation of the promoter region. Mol Cell Biol, 16, 276-43.

Brewer CF (2004). Thermodynamic binding studies of galectin-1, -3 and -7. Glycoconj, 19, 459-65.

Biron-Pain K, Grosset AA, Poirier F, Gaboury L, St-Pierre Y (2013). Expression and functions of galectin-7 in human and murine melanomas. PLoS One, 8, 63307.

Cada Z, Chovanec M, Smetana K, et al (2009). Galectin-7: will the lectin's activity establish clinical correlations in head and neck squamous cell and basal cell carcinomas? Histol Histopathol, 24, 41-8.

Campion CG, Labrie M, Lavoie G, St-Pierre Y (2013). Expression of galectin-7 is induced in breast cancer cells by mutant p53. PLoS One, 8, 72468 .

Campion CG, Labrie M, Grosset AA, St-Pierre Y (2014). The CCAAT/enhancer-binding protein beta-2 isoform (CEBP $\beta$-2) upregulates galectin-7 expression in human breast cancer cells. PLoS One, 9, 95087.

Chen J, He QY, Yuen AP, Chiu JF (2004). Proteomics of buccal squamous cell carcinoma: the involvement of multiple pathways in tumorigenesis. Proteomics, 4, 2465-75.

de Vasconcelos Carvalho M, Pereira Jdos S, Alves PM, et al (2013). Alterations in the immunoexpression of galectins-1, -3 and -7 between different grades of oral epithelial dysplasia. J Oral Pathol Med, 42, 174-9.

Demers M, Magnaldo T, St-Pierre Y (2005). A novel function for galectin-7: promoting tumorigenesis by up-regulating MMP-9 gene expression. Cancer Res, 65, 5205-10.

Demers M, Biron-Pain K, Hébert J, et al (2007). Galectin-7 in lymphoma: elevated expression in human lymphoid malignancies and decreased lymphoma dissemination by antisense strategies in experimental model. Cancer Res, 67, 2824-9.

Demers M, Couillard J, Giglia-Mari G, Magnaldo T, St-Pierre Y (2009). Increased galectin-7 gene expression in lymphoma cells is under the control of DNA methylation. Biochem Biophys Res Commun, 387, 425-9.

Demers M, Rose AA, Grosset AA, et al (2010). Overexpression of galectin-7, a myoepithelial cell marker, enhances spontaneous metastasis of breast cancer cells. Am J Pathol, 176, 3023-31.

Duray A, De Maesschalck T, Decaestecker C, et al (2014). Galectin fingerprinting in naso-sinusal diseases. Oncol Rep, 32, 23-32.

George J, Srivastava AK, Singh R, Shukla Y (2011). Cypermethrin exposure leads to regulation of proteins expression involved in neoplastic transformation in mouse skin. Proteomics, 11, 4411-21.

Grosset AA, Labrie M, Gagné D, et al (2014). Cytosolic galectin-7 impairs p53 functions and induces chemo resistance in breast cancer cells. BMC Cancer, 14, 801 .

Hughes RC (1999). Secretion of the galectin family of mammalian carbohydrate-binding proteins. Biochim Biophys Acta, 1473, 172-85.

Kim HJ, Jeon HK, Lee JK, et al (2013a). Clinical significance of galectin-7 in epithelial ovarian cancer. Anticancer Res, 33, 1555-61.

Kim SJ, Hwang JA, Ro JY, Lee YS, Chun KH (2013b). Galectin-7 is epigenetically-regulated tumor suppressor in gastric cancer. Oncotarget, 4, 1461-71.

Labrie M, Vladoiu MC, Grosset AA, Gaboury L, St-Pierre Y (2014). Expression and functions of galectin-7 in ovarian cancer. Oncotarget, 5, 7705-21.

Leffler H, Carlsson S, Hedlund M, Qian Y, Poirier F (2004). Introduction to galectins. Glycocon, 19, 433-40.

Liu FT, Rabinovich GA (2005). Galectins as modulators of tumour progression. Nat Rev Cancer, 5, 29-41.

Lu J, Pei H, Kaeck M, Thompson HJ (1997). Gene expression changes associated with chemically induced rat mammary carcinogenesis. Mol Carcinog, 20, 204-15.

Magnaldo T, Bernerd F, Darmon M (1995). Galectin-7, a human 14-kDa S-lectin, specifically expressed in keratinocytes and sensitive to retinoic acid. Dev Biol, 168, 259-71.

Magnaldo T, Fowlis D, Darmon M (1998). Galectin-7, a marker of all types of stratified epithelia. Differentiation, 63, 159-68.

Matsui Y, Ueda S, Watanabe J, Kuwabara I, Ogawa O, Nishiyama $\mathrm{H}$ (2007). Sensitizing effect of galectin-7 in urothelial cancer to cisplatin through the accumulation of intracellular reactive oxygen species. Cancer Res, 67, 1212-20.

Matsukawa S, Morita K, Negishi A, et al (2014). Galectin-7 as a potential predictive marker of chemo- and/or radio-therapy resistance in oral squamous cell carcinoma. Cancer Med, 3, 349-61.

Meng QS, Jin S, Zhang QH, Zhang M (2010). Differential proteins analysis among human nasal inverted papilloma and nasal polyposis and normal nasal mucosa. Zhonghua Er Bi Yan HouTou Jing Wai Ke Za Zhi, 45, 314-7. 
Moisan S, Demers M, Mercier J, et al (2003). Upregulation of galectin-7 in murine lymphoma cells is associated with progression toward an aggressive phenotype. Leukemia, 17, 751-9.

Newlaczyl AU, Yu LG (2011). Galectin-3--a jack-of-all-trades in cancer. Cancer Lett, 313, 123-8.

Ostergaard M, Rasmussen HH, Nielsen HV, et al (1997). Proteome profiling of bladder squamous cell carcinomas: identification of markers that define their degree of differentiation. Cancer Res, 57, 4111-7.

Park JE, Chang WY, Cho M (2009). Induction of matrix metalloproteinase- 9 by galectin-7 through p38 MAPK signaling in HeLa human cervical epithelial adenocarcinoma cells. Oncol Rep, 22, 1373-9.

Polyak K, Xia Y, Zweier J, Kinzler K, Vogelstein B (1997). A model for p53-induced apoptosis. Nature, 389, 300-5.

Rorive S, Eddafali B, Fernandez S, et al (2002). Changes in galectin-7 and cytokeratin-19 expression during the progression of malignancy in thyroid tumors: diagnostic and biological implications. Mod Pathol, 15, 1294-301.

Ruebel KH, Jin L, Qian X, et al (2005). Effects of DNA methylation on galectin-3 expression in pituitary tumors. Cancer Res, 65, 1136-40.

Satelli A, Rao PS, Gupta PK, et al (2008). Varied expression and localization of multiple galectins in different cancer cell lines. Oncol Rep, 19, 587-94.

Sato M, Nishi N, Shoji H, et al(2002). Quantification of galectin-7 and its localization in adult mouse tissues. $J$ Biochem, 131, 255-60.

Saussez S, Kiss R (2006). Galectin-7. Cell Mol Life Sci, 63, 686-97.

Saussez S, Cucu DR, Decaestecker C, et al (2006). Galectin 7 (p53-induced gene 1): a new prognostic predictor of recurrence and survival in stage IV hypopharyngeal cancer. Ann Surg Oncol, 13, 999-1009.

Saussez S, Decaestecker C, Lorfevre F, et al (2008a). Increased expression and altered intracellular distribution of adhesion/ growth-regulatory lectins galectins- 1 and -7 during tumour progression in hypopharyngeal and laryngeal squamous cell carcinomas. Histopathology, 52, 483-93.

Saussez S, Lorfevre F, Lequeux T, et al (2008b). The determination of the levels of circulating galectin- 1 and -3 in HNSCC patients could be used to monitor tumor progression and/or responses to therapy. Oral Oncol, 44, 86-93.

Saussez S, Cludts S, Capouillez A, et al (2009). Identification of matrix metalloproteinase- 9 as an independent prognostic marker in laryngeal and hypopharyngeal cancer with opposite correlations to adhesion/growth-regulatory galectins-1 and -7. Int J Oncol, 34, 433-9.

Shen J, Riggs PK, Hensley SC, et al (2007). Differential expression of multiple anti-apoptotic proteins in epidermis of IGF-1 transgenic mice as revealed by 2-dimensional gel electrophoresis/mass spectrometry analysis. Mol Carcinog, 46, 331-40.

Shen J, Pavone A, Mikulec C, et al (2007). Protein expression profiles in the epidermis of cyclooxygenase- 2 transgenic mice by 2 -dimensional gel electrophoresis and mass spectrometry. J Proteome Res, 6, 273-86.

St-Pierre Y, Grosset AA, Labrie M, et al (2015). Galectin-7 increases resistance of breast cancer cells to drug-induced apoptosis and promotes tumor escape by killing $\mathrm{T}$ cells Cancer Res, 75, 7-16.

Tsai CJ, Sulman EP, Eifel PJ, et al (2013). Galectin-7 levels predict radiation response in squamous cell carcinoma of the cervix. Gynecol Oncol, 131, 645-9.

Than TH, Swethadri GK, Wong J, et al (2008). Expression of Galectin-3 and Galectin-7 in thyroid malignancy as potential
DOI:http://dx.doi.org/10.7314/APJCP.2016.17.2.455

Expression and Roles of Galectin-7 in Different Types of Cancer diagnostic indicators. Singapore Med J, 49, 333-8.

Ueda S, Kuwabara I, Liu FT (2004). Suppression of Tumor Growth by Galectin-7 Gene TransferGalectin-7 levels. Cancer Res, 64, 5672-6.

Vladoiu MC, Labrie M, St-Pierre Y (2014). Intracellular galectins in cancer cells: potential new targets for therapy (Review). Int J Oncol, 44, 1001-14.

Zhu H, Pei HP, Zeng S, et al (2009). Profiling protein markers associated with the sensitivity to concurrent chemoradiotherapy in human cervical carcinoma. $J$ Proteome Res, 8, 3969-76.

Zhu H, Wu TC, Chen WQ, et al (2013). Roles of galectin-7 and S100A9 in cervical squamous carcinoma: Clinicopathological and in vitro evidence. Int J Cancer, 132, 1051-9.

Zhu X, Ding M, Yu ML, et al (2010). Identification of galectin-7 as a potential biomarker for esophageal squamous cell carcinoma by proteomic analysis. BMC Cancer, 10, 290. 